\title{
Corrigenda for Holomorphic Flows on Simply Connected Regions have No Limit Cycles
}

\author{
Kevin A. Broughan
}

\author{
University of Waikato, Hamilton, New Zealand \\ E-mail: kab@waikato.ac.nz
}

There is an error in Theorem 4.1 in [1] which was very kindly pointed out to me by Deming Liu, the last part of the last sentence being incorrect (as shown by Example 4.2 in case $n=3$ ). The correct statement and revision for the proof are given here.

ThEOREM 0.1. Let $\dot{z}=f(z)=\alpha\left(z-z_{1}\right) \cdots\left(z-z_{n}\right)$ be a flow where $\alpha \in \mathbb{C} \backslash\{0\}$, the $z_{i}$ are distinct, and each is a center (for the linearized flow). If $n \leq 3$ then the $z_{i}$ are collinear. If $n=4$ then the $z_{i}$ are either collinear or any three form the vertices of a triangle with the remaining point the orthocenter of the triangle.

Proof. If $n \leq 3$ the proof is the same as given in [1, Theorem 4.1]. If $n=$ 4 the proof is the same, until the very last sentence, which should read: But then $z_{1}-z_{3} \perp z_{4}-z_{2}, z_{1}-z_{2} \perp z_{3}-z_{4}$ and $z_{1}-z_{4} \perp z_{3}-z_{2}$, so any three of the points form a proper triangle for which the remaining point is the orthocenter.

\section{REFERENCES}

1. Broughan, K.A. Holomorphic flows on simply connected regions have no limit cycles, Meccanica 38 (2003), p699-709. 